\title{
The Ecosystem of the Next-Generation Autonomous Vehicles
}

\author{
Saleem Sahawneh ${ }^{*}, 1$, Ala' J. Alnaser ${ }^{2}$ \\ ${ }^{1}$ Computer Science Department, Florida Polytechnic University, Lakeland, FL 33805-8531, USA \\ ${ }^{2}$ Department of Applied Mathematics, Florida Polytechnic University, Lakeland, FL 33805-8531, USA
}

\begin{tabular}{l} 
A R T I C L E I N F O \\
Article history: \\
Received: 21 November, 2020 \\
Accepted: 16 January, 2021 \\
Online: 25 February, 2021 \\
\hline Keywords: \\
Autonomous vehicles \\
Analysis of AV accidents \\
AV Eco-System Infrastructure \\
Recommendations \\
Event data recorder
\end{tabular}

\section{Introduction}

\subsection{Conventional Automobile Regulation}

There is a desire everywhere to reduce the number of car accidents on our roads due to its effects on the communities and the economy. This paper is an extension of work originally presented in [1] in which we studied a number of autonomous vehicle's accidents and analyzed them in order to understand the behaviour of autonomous vehicles and to locate the root causes of these accidents. Studying traffic accidents is not new. Actually, accidents have been a concern for societies since the first recorded fatality back in 1869 [2]. There are different causes of accidents such as the weather, obstructions on the roads, mechanical problems with the vehicles, etc. However, the main causes are the drivers themselves who might be drowsy, intoxicated or over speeding [3]-[4]. Problems of traffic congestion and road accidents have increased due to the increase in the demands of vehicles [5]. Nowadays, over a million people lose their lives due to road traffic crashes world wide and these

\footnotetext{
${ }^{*}$ Corresponding Author: Saleem Sahawneh, Email: ssahawneh@ floridapoly.edu
}

crashes cost most countries $3 \%$ of their gross domestic product [6]. Some advanced driving assistance systems helped in improving the safety of vehicles on the roads such as Adaptive Cruise Control. The current goal that manufactures are working on now is to have fully automated vehicles on roads within the foreseeable time and this will reduce the number of accidents significantly on the roads [7]. These autonomous vehicles are in their experimental phase where they operate on the roads along with a backup (safety drivers) to take control as needed and to avoid accidents.

In order to manage automobile accidents, one must consider a number of involved parties, such as local law enforcement, insurance companies, state licensing authorities and federal authorities [1]. The driving process can be divided into three aspects: Perception, Decision Making and Action. Currently, the human driver operating a traditional vehicle is responsible of the perception as well as the decision making of the driving process. The manufacturer of the vehicle is responsible for the action aspect of the driving process. When an accident occurs, an accident report is produced by the law enforcement who are the first engagement entity in the 
creation of the accident report. Law enforcement investigate the accident and includes the following information:

- Location and timing of the accident.

- Identifying information of drivers and vehicles.

- Description of the accident based on drivers, passengers, and other witnesses.

- Description of property damages and medical issues.

- Occasionally, weather conditions are included

The investigation of the accident determines the direct cause of the accident and determines which party is at fault. The fault can be related to drivers or the vehicle itself. Then the other entities, including insurance companies, lawyers and court system, would intervene to solve the financial consequences of the accident. However, if the vehicle itself is at fault then the National Highway Traffic Safety Administration (NHTSA) gets involved [8]. One of the main tasks of NHTSA is to license vehicles, offer feedback on safety of vehicles and to locate systemic vehicle issues and fix them The Crash Investigation Sampling System (CISS) or the Office of Defects Investigation (ODI) are usually the initiators of the NHTSA investigations.

It is note worthy that the Federal Aviation Administration (FAA) has been very successful in their accident investigation methods where there is a requirement to include a flight data recorder (FDR) and a cockpit voice recorder (CVR) inside what is referred to as "a black box". Besides that, safety concerns are confidentially gathered for further analysis.

Vehicles have similar technology to the FDR, namely, the Event Data Recorder (EDR). The EDR is a device installed in a motor vehicle to record technical vehicle and occupant information for a brief period of time before, during and after a crash [9]. However, it is not required by the Federal Government to install it in all vehicles [10] The official definition of the EDR and the minimal list of parameters that must be recorded was produced by the NHTSA in 2019. The recorded parameters include longitudinal and latitudinal speeds and accelerations, engine throttle and RPM, time, ABS activity, air bags deployment status, role angle of the vehicle, occupants position and many more which are recorded until a fraction of a second before an event or accident [11]. Furthermore, EDR technology has been voluntarily used with almost $92 \%$ of new light automobiles since 2012.

\subsection{AV Accident Reports}

The traditional driving system assumes that the perception and decision making aspects of the driving process are the responsibility of the human driver. However, with an AV system, the responsibility for perception and decision making is shifted to the vehicle itself. This transition from humans to AVs requires developing new procedures and laws (regulations) that can answer some of the new challenging questions such as how to determine who owns liability in case of an accident, how to license AVs, and how NHTSA can certify a fleet of AVs.
In the United States, a number of local states authorities have adopted different laws to regulate $\mathrm{AV}$ as a driver. For example, California's Department of Motor Vehicle (DMV) adopted regulations for testing AVs in 2014. In a followup amendment in 2018 [12], the laws requires the manufacturers to submit an AV collision report within a period 10 days of any accidents (minor or major). The report includes the following information: Manufacturer's information, cars involved (time, location of the accident, description of damage, etc. ), property damage, a description of the accident, a check list that contains information about the weather, lightning, road way surface (wet, ery, etc) and road way conditions (flooded, holes, etc). Moreover, for each vehicle involved in the collision, the report contains information about movement preceding collision (stopped, backing, etc), type of collision (head-on, side swipe, etc) and associated factors (such as vision obstruction).

Besides the collision reports, every manufacturer is required to submit an annual report, by January 1st of each year, that contains all the disengagements of the AV technology during testing their AVs on California's public roads. These reports show how often the vehicles were disengaged from autonomous mode during test. The disengagements could be because of technology failures or simply because the backup driver wanted to safely operate the vehicle.

Beyond California, none of the other states provide guidance on AV accident reports.

\subsection{Related Work}

Different aspects related to Autonomous vehicle's accidents and safety have been studied such as Collision Avoidance [13], the ethical decision-making during crashes [14] and the Automated Vehicles Safety in Lane-Change Scenarios [15]. However, studies of real AV's crashes have been limited to collecting and analyzing AV crash data from a limited number of accident's reports [16], [17]. There is an agreement between the researchers in this field that the current AV crash reports are unstructured and sparse which results in impeding the learning and analysis work. The importance of the information in the accident reports help in capturing systemic problems that might exist even in what seems to be minor accidents.

In [1], a database was built using reports collected on $94 \mathrm{mi}-$ nor and major accidents involving AVs. The data was statistically analyzed and that helped in building a foundation for a proposed accident report that meets the AV needs. In this work, we expanded the database to include 200 accidents and the analysis has been performed on all of these accidents.

This paper focuses on suggestions and mechanisms to determine and capture the necessary data that is needed in order to capture any systemic issues. This is important in the verification of AVs where the accidents can be simulated by creating the same scenarios which led to the accidents. This is helpful to determine if the decision made by the AV involved in the accident was as expected or not. The real crash data is valuable with this regard.

This paper follows the following outline; Section 2 describes the core architecture of AV systems. The analysis of current crashes from the point-of-view of developing a safety methodology is in Section 3. Our recommendations for an improved AV ecosystem infrastructure is presented in Section 4 and finally, Section 5, offers conclusions. 


\section{The AV system Architecture}

The key to understanding the reasons behind the accidents that involve $\mathrm{AVs}$ is understanding the structure of the systems that the $\mathrm{AV}$ is comprised of. As mentioned briefly before, the AV operates using the three standard stages of driving: Perception, Decision Making and Action. Figure 1 presents the major components of the AV system where the components are divided with respect to their contribution to each of the driving stages. When a human driver is in control of a traditional vehicles, the perception stage and decision making stage are the responsibility of the driver, while the action is performed by the vehicle. The automotive industry has over 100 years of experience in making certain that the action is performed in an optimum manner. Comparatively, the perception phase through the use of sensors is very recent. Sensing the environment is accomplished using sensors such as cameras, radar and lidar technologies. All the inputs of these components are combined with object recognition artificial intelligence engines. Perception also combines the sensory input with communication from other vehicles (V2V) and the surrounding infrastructure (V2I). The internal model of the surrounding environment of the AV is finally created by combining all these inputs.

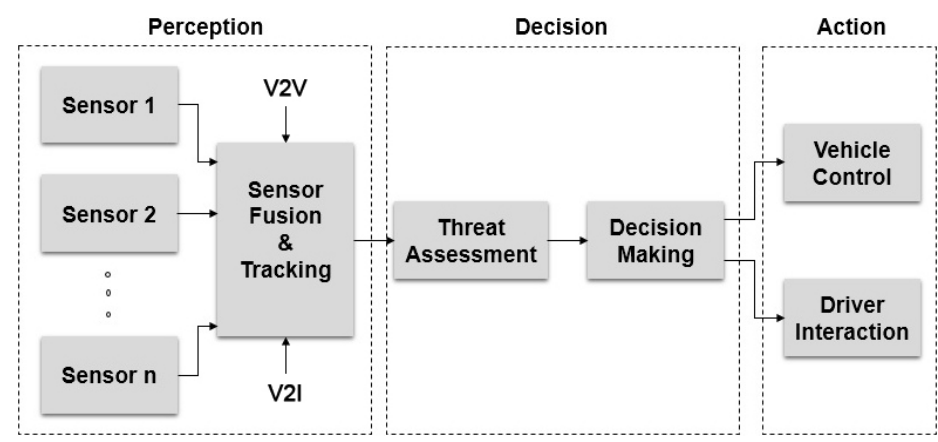

Figure 1: Autonomous vehicle technology architecture

In the context of reports on accidents involving AV's, the gathering of sufficient amount of information is imperative so that it can be analyzed to understand what the AV perceived and the reason behind the decision it made. Looking at the architecture of AVs, when an accident occurs, several open questions arise. These include:

- Are the sensors functioning as required and as intended ?

- What is the accuracy level of the the sensor fusion/object recognition?

- Is the threat assessment and the analysis of any obstruction correct?

- Assuming perfect input, is the AV making the correct decision?

- What is the accuracy of the internal maps?

- Is the communication between the vehicle and the environment (V2I) and between the vehicle and other vehicles (V2V) understood properly?

We will now examine whether we can diagnose these issues with current information.

\section{Analysis of Current Accidents}

An analysis of all reported $\mathrm{AV}$ accidents up to the present date was carried out with the objective being to understand the needs of future accident reports regardless of the automation level at this point These AVs are being tested on the roads and they require a safety driver while the AVs perform driving.

\subsection{Data collection}

After mining the public records of the Department of Motor Vehicles in California in addition to multiple sources from the press, a database of accidents involving AVs was built. The database included details in the following categories:

- Information about the road and environment

- The location of the accident using GPS coordinates

- Atmospheric and weather conditions

- Types of the roads and road structure (intersections, high ways, etc)

- Information about the accident

- Description of the traffic at the time of the accident

- Manner of accident (rear-ending, side swiped, etc)

- Date and time of the incident

- The speeds of the involved vehicles

- The road speed limit

- Severity/fatality level of accident

- Information about the AV

- The manufacturing company operating the AV

- Vehicles' models

- The autonomous mode used during the incident

As faced by other researchers in this area, converting the unstructured loose textual data into formats which would enable analysis on a deeper level is vital but quite challenging. The database was populated with all the information that was available, however, the data was incomplete in many cases, specially in the cases of minor accidents. To fill in some of the missing information, such as the type of roads and number of lanes, Google Earth was used based on the location of accidents provided in the accident reports (location of an accident is given as an address or as a cross street in the report). 


\subsection{Detailed review of minor accidents}

The earliest record we have of an accident involving an autonomous vehicle dates back to May of 2010, since then there has been many other accidents. However, getting access to up-to-date information and records of these accidents to create a database has been quite challenging. We have looked and gathered information on about 200 accidents with 189 of these accidents in the state of California. That is due to the fact that the state of California was one of the first states to put into place a law that allows the AVs to be tested on public roads and that allows police officers to write accident reports for all accidents involving AVs and make these reports available as public records. In addition, most of the manufacturers and Technology Developers are based in California and they were testing vehicles close to their facilities. In Figure 2, is a list of all manufacturers and developers we have in our database and their percentages of accidents. As anticipated, manufacturers and developers with larger fleets of AVs, such as GM Cruise and Google (Waymo), have had bigger share of accidents with $46 \%$ and $37 \%$ (combined) respectively. It is also worth noting that the number of companies investing and working in this field has increased in the last 2 years [1].

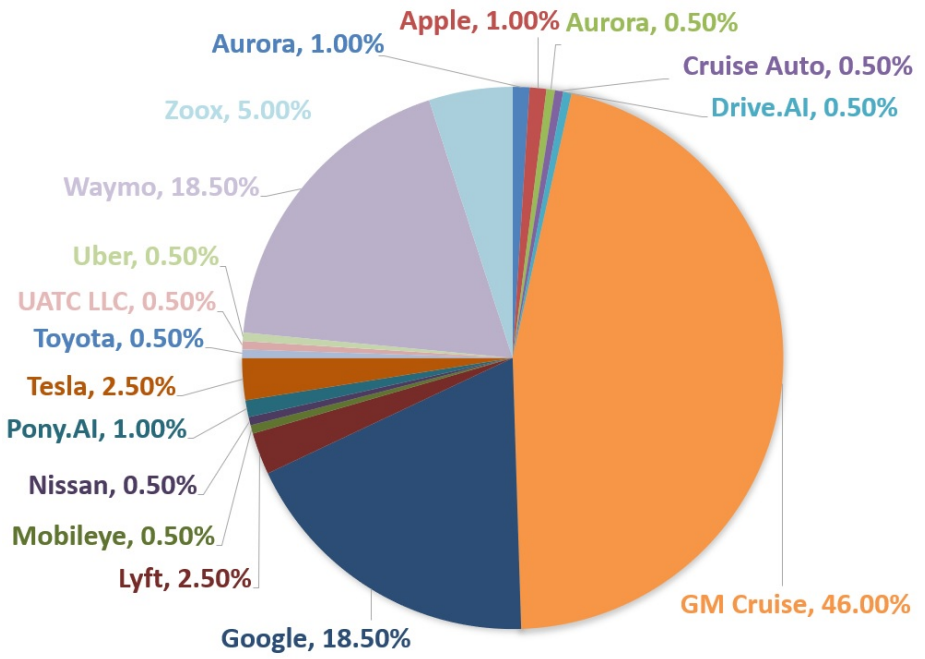

Figure 2: Percentages of crashes by AV Developers

The accident reports were not consistent, and in many cases the reports were missing many pieces of information which are necessary to truly understand what caused the accident and all the parameters that were involved.

There are many characteristics of an accident that will be much more important now that AVs are on the roads performing the perception, decision making, and the mechanics of the driving process. Characteristics such as speeds of all vehicles involved in the accident and the speed limit, as well as the road structure, just to name a few, are specially important, to be able to simulate the accidents accurately and understand the cause. Even minor accidents could be caused by a systemic error in the decision making algorithms which could cause much bigger issues later. However, many of these characteristics are either missing or not required by the current accidents report forms.

In the construction of our database, much effort and time was spent analyzing accident reports and filling in missing information. We have found that the AV speed was unknown in $51 \%$ of the accidents, $63.5 \%$ of the accidents speed of the second elements in the crash (vehicle or object) was not listed. In $83.5 \%$ of the accidents, the speed limit for the road on which the accident happened was not recorded. Also, the type of road on which the accident occurred was not clear in $6.25 \%$ of the reports.

Figure 3, illustrates the accidents sorted by road type as listed on the accidents' reports. The majority of the accidents happened at intersections, especially at four-way intersections where $68.5 \%$ of all the crashes occurred. These accidents could be caused by a failure of the sensors or the algorithms which are supposed to gather sensor input and fuse it together in order for the $\mathrm{AV}$ to have an accurate model of the environment around it. Furthermore, it is worth noting that the environment at intersections is quite complex.

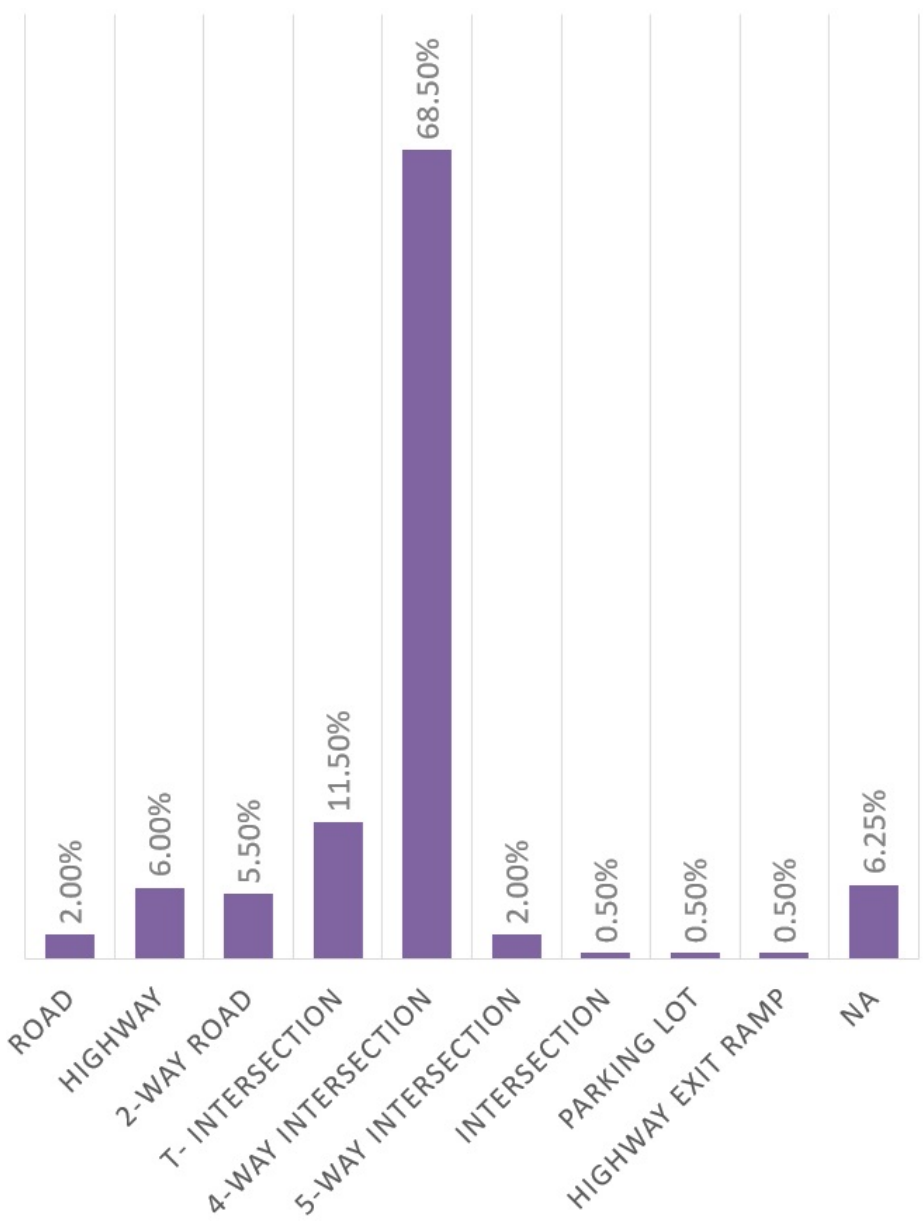

Figure 3: Types of roads where accidents happened

The chart in Figure 4 shows the speed ranges of vehicles (elements) involved in the crashes and the percentage of crashes that happened in these ranges. As mentioned previously, the speeds for $\mathrm{AV}$ and the other element or vehicle was not recorded in many reports. However, out of the ones that were recorded we observed that the majority of crashes happened at low to idling speeds. This was not surprising since the vast majority of accidents happened at intersections where the vehicles were either stopping or just starting to move from a full stop. In addition, AV testing has been limited to nice weather and low speed situations. 


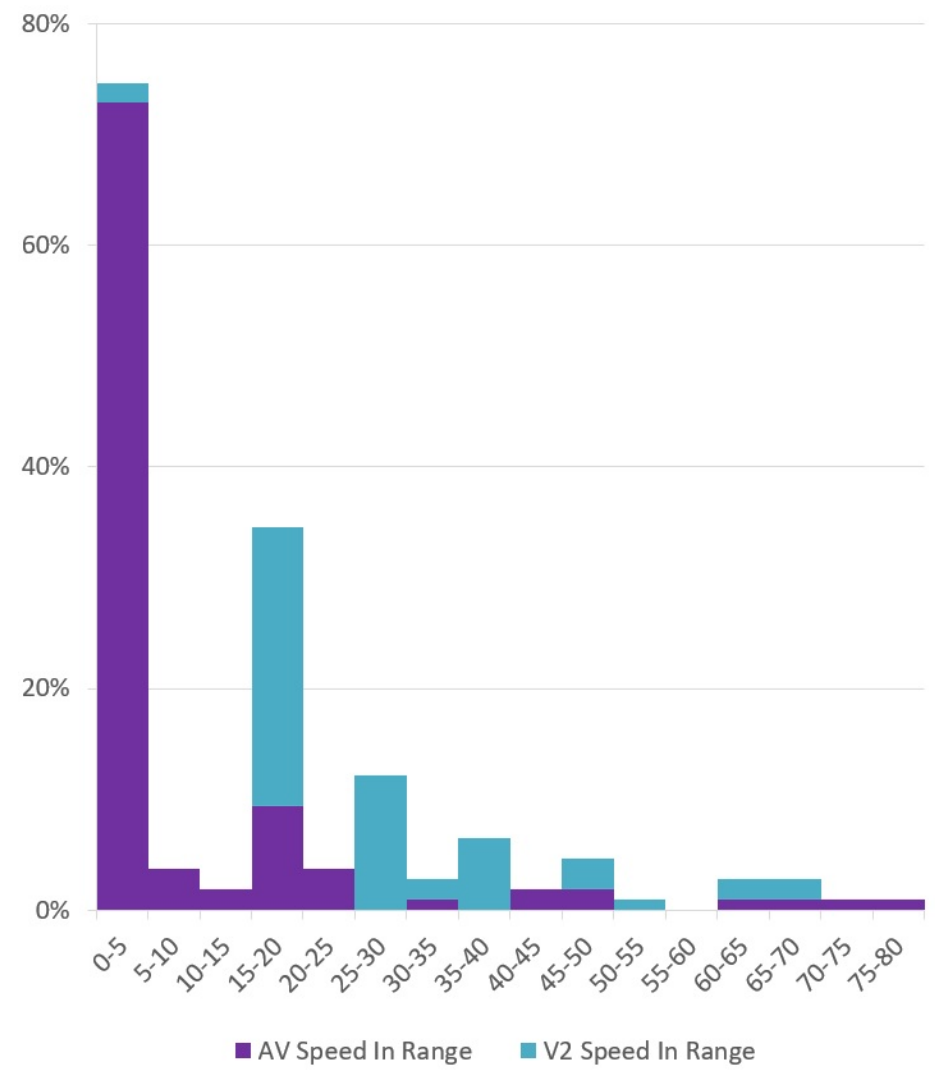

Figure 4: The percentage of accidents corresponding to speed range

Table 1: Accidents types and percentages and the status of the AV system.

\begin{tabular}{|l|c|c|}
\hline \multirow{2}{*}{ Type of Accident } & \multicolumn{2}{|c|}{ AV Mode } \\
\cline { 2 - 3 } & Engaged & Disengaged \\
\hline Taxi Driver slapped a window & $0.0 \%$ & $1.3 \%$ \\
\hline A vehicle backed into AV & $0.0 \%$ & $1.3 \%$ \\
\hline A vehicle rear ended the AV & $67.8 \%$ & $38.0 \%$ \\
\hline Angle & $9.1 \%$ & $7.6 \%$ \\
\hline A vehicle struck the AV's rear bumper & $0.0 \%$ & $1.3 \%$ \\
\hline AV Backed into another vehicle & $0.0 \%$ & $1.3 \%$ \\
\hline AV rear ended another car & $0.8 \%$ & $2.5 \%$ \\
\hline Broadside & $2.5 \%$ & $7.6 \%$ \\
\hline Head-on & $0.0 \%$ & $5.1 \%$ \\
\hline Hit by Pedestrian & $0.8 \%$ & $1.3 \%$ \\
\hline Hit Object & $0.8 \%$ & $3.8 \%$ \\
\hline Perpendicular & $1.7 \%$ & $3.8 \%$ \\
\hline Rear end, Head on & $0.0 \%$ & $2.5 \%$ \\
\hline Side swipe & $9.1 \%$ & $22.8 \%$ \\
\hline A car made slight contact AV's side & $0.8 \%$ & $0.0 \%$ \\
\hline A pedestrian struck. & $0.8 \%$ & $0.0 \%$ \\
\hline Radar Clipped by a passing truck & $0.8 \%$ & $0.0 \%$ \\
\hline AV Front to back of firetruck & $0.8 \%$ & $0.0 \%$ \\
\hline Rear ended a fire truck & $0.8 \%$ & $0.0 \%$ \\
\hline The AV crossed the red light & $0.8 \%$ & $0.0 \%$ \\
\hline NA & $2.5 \%$ & $0.0 \%$ \\
\hline
\end{tabular}

It was surprising to find that the vast majority of AV accidents are actually humans hitting AVs (see Table 1). Furthermore, when also considering the type of accident and speeds at which the accidents happen, one may conjecture that a portion of these accidents could have happened because of a lack of understanding of the behavior of the AV. For instance, $67.8 \%$ of the crashes were involving another vehicle rear-ending the $\mathrm{AV}$ while it was slowing down to turn right at an intersection. The AV's are required to make a full stop before turning right when there is a red light or a stop sign while the human driver in the vehicle behind it might have predicted that the AV will just slow down and make the turn.

\subsection{Major accidents}

Autonomous Vehicle's major accidents with severe damage, injury or fatalities occurrence are very important to analyze. The information obtained from any accident can be used to understand the behavior (actions) of AVs under different circumstances. In [1], three fatal accidents were analyzed. These accidents took place in three different states in USA and were reported in media: Florida, California and Arizona between 2016 and 2018. Each major accident happened under different scenarios from the other ones. For instance, the autonomous vehicles were driving at different speeds ranging from 43 up to $74 \mathrm{mph}$ at the time of the accident. However, we noticed one common thing between these accidents which is that the object (truck, road barrier or a pedestrian) that the AVs collided with was either stopping or moving at very low speed.

Upon analyzing these major accidents, we found out the following findings:

- Autonomous Vehicles, in some cases, were able to sense the objects in front of them few seconds before the accident but did not recognize it as a threat. For example, the software on Uber's AV system, back in 2018, initially classified the pedestrian crossing the road in front of Uber as an another vehicle moving in the same direction before reclassifying the pedestrian as a bicycle that was static.

- Some procedures for risk assessment were not appropriate including oversight of the backup drivers.

- Threat analysis is a critical in the development of AVs.

- When the autopilot is used to follow a leading car, while navigating other traffic and road curvature conditions, there is a need for continuous perception and a stable feedback system with the leading car under all conditions. If that feedback is interrupted then a back up plan should be implemented.

- Autonomous vehicles, under experimental driving, issue warnings to the human back up drivers in some situations. An action plan must be implemented when the human driver ignore these warnings. For example, in Uber accident, The National Transportation Safety Board (NTSB) determined that one of the main causes of the accident was the failure of the Uber backup driver to monitor the road.

- There is a lack of system prevention measures to prevent the possibility of misuses of technology by the drivers specially when drivers lack the understanding of the limitations of the autopilot. 
- There is a need to record and take instant action when complains are received from the users of the vehicles because that could reveal a systemic problem. As an example, in California's Tesla accident, the driver had complained few times that his Model X vehicle would drift of its lane in the same location where the collision occurred while using the autopilot.

- Manufacturers should be involved in order to get an independent analysis and verification of the data associated with the vehicle's crashes. Also, the data must be standardized and retrievable. This retrieved information is extremely important to detect if an issue is an indication of a systemic risk.

In 2019, another fatal major accident took place in Florida, USA and it is discussed in this paper.

Details of Accident: On March 1, 2019, about 6:17 a.m, A fatal crash occurred when a 2018 Tesla Model 3 under-rode a truck (truck-tractor in combination with a semitrailer) which resulted in the death of the Tesla driver. This accident took place in State Highway 441 in Delray Beach, Florida. The accident happened when Tesla, driving southbound at $68 \mathrm{mph}$ on a $55 \mathrm{mph}$ speed limit road, came across a truck that pulled out of a private driveway on the right hand side of the road and blocked Tesla's path. The NTSB's preliminary report stated that the truck slowed down as it crossed Tesla's lane and blocked Tesla's path. The truck was trying to make a left turn into northbound lanes and Tesla's ADAS didn't execute an evasive maneuvers to stop or change direction of the car. The car came to a stop on the highway's earthen median, about 1,600 feet away [18]. Besides that, the NTSB report states that the driver of Tesla engaged the autopilot 10 seconds before the impact and that the driver's hands weren't detected on the steering wheel from less than 8 seconds to the crash. This accident reminds us of the 2016 Tesla crash that took place in Florida. Both of the accidents involved tractor-trailer trucks with similar scenarios and both resulted in the unfortunate death of the drivers. In both accidents, the autopilots were engaged.

The accident in 2016 took place when a semi-autonomous Tesla Model S70D collided with a tractor-trailer while approaching an intersection. The tractor was making a left turn across the path of the Tesla, but Tesla did not stop and crashed into the trailer [1]. Tesla was using a black and white camera and there is a possibility that the computer was believed incorrectly that the road in front of the Tesla was clear. This accident might have been avoided if a LIDAR sensor was used instead of the camera since the colors would not have mattered [19]. Also, the autopilot system was tuned to ignore more of the radar inputs at high speed in order to avoid false positives.[20].

Similarities of both accidents: Despite the time difference between the two accidents and also despite the software updates that have been applied to the autopilot (AP1 to AP2) since the first accident [21], there are major similarities between the two accidents: both of accidents involved hitting stationary truck-trailors at high speed and the semi-autonomous vehicles didn't attempt to stop. Also, both of the accidents happened in sunny and clear skies days.

These two accidents show that a safety challenge still exists with the autopilots and how they deal with stationary objects at high speeds. Correct AV's perception of the environment is critically im- portant for safety. In the above mentioned crashes, it seems that the autopilot failed to perceive the truck-trailors as objects blocking the path of the AV. On the other hand, a false perception could also lead to dangerous accidents. For example, an $\mathrm{AV}$ mistakenly perceives a moving object as a stationary object. This situation could trigger the autopilot to stop the car completely and that is a dangerous action specially if the vehicle is travelling at high speed on the freeway which might cause other vehicles to rear end the AV.

These accidents highlight the need to understand the perception aspect of the AV. If the perception engine is fooled then that might lead to accidents. The communication between vehicles themselves on the road (vehicle to vehicle, $\mathrm{V} 2 \mathrm{~V}$ ) and also with the infrastructure (V2I) might be necessary to avoid accidents. Also, it might be useful to use more accurate sensors such as LIDARS instead of cameras in order to reduce the risk of misconception by the vehicle.

Analysing the accident and understanding why the AV took the decision is extremely important to locate the problem and work on solving it to prevent it from happening again in the future. Therefore, the data collected from these accidents are important.

\section{Recommendations on AV EcoSystem In- frastructure}

The creation of an ecosystem or road environments in which AVs can operate at a higher level of efficiency requires upgrades in three major areas. This is based on analysing the accidents up to the present date and the methods by which information was gathered, as well as, the operational fundamentals of AVs. The areas in need of improvement are:

- Utilization of data from Event Data Recorders (EDRs) for vehicles and infrastructure

- Update and supplement Vehicle Accident Report

- Upgrade Fatality Analysis Reporting System (FARS) and an Automated Storage Retrieval System (ASRS) system

\subsection{EDR Information}

As mentioned earlier, Event Data Recorders are currently available in over $92 \%$ of vehicles on the road. However, EDRs are needed as a requirement for all AVs. Regardless of the model, an EDR collects and record the current vehicle dynamics. However, an EDR in an AV (AV-EDR) need to capture additional AV critical data. The AV critical data include raw sensor inputs, all communications ( $\mathrm{V} 2 \mathrm{~V}$ and $\mathrm{V} 2 \mathrm{I}$ ), the ongoing object model of the surrounding environmental produced by the perception stage, and finally the threat annotations of the object model.

The time-based unmodified recordings of all the sensors (Camera, Lidar, Radar, etc) together with the vehicle dynamics produce the mathematical model for the AV. Using this mathematical model and information, issues or faults related to manufacturing, electromagnetic interference, or weather can be detected.

One of critical components for understanding faults of object recognition is details and accurate recording of the perceived object model and its corresponding threat annotations. The core AI sitting 
at the center of the AV systems are possibly the most challenging to construct and test. If the AI system has a fundamental error, then it is likely that this error will reveal itself in other circumstance. Therefore, such errors can give rise to systemic risks beyond the current incident.

Moreover, recording all the communication between the vehicles and with the environment (V2V and V2I) information is essential to identify issues in the case where communication was planned but failed. Also, to determine if errors occurred due to the actual message contained within the communication is faulty or if communication is compromised.

Finally, AV-EDR data should be recorded locally and on the cloud in standard formats. The combination of EDR recordings for the AV and the environment form a reasonably complete package information for any accident of that is automatically collected. In the case of an accident, law enforcement at the scene should have instant access to the EDR information.

\subsection{AV Police Accident Report}

The current accident report procedure has been optimized to handle human related traffic accidents. However, in order to deal with this upcoming technology, the accident procedure needs to be updated in three main areas; severity, the interview with the driver, and in terviews with other humans. For severity, since the human beings behaviour is well understood, current minor accidents are treated lightly. On the other hand, when an AV is involved in minor incidents it could be an indication of a systematic fault or safety issues that may cause major accident in the future. Hence, law enforcement will need to put additional focus on all accidents involving $\mathrm{AVs}$ even the minor accidents.

Normally, law enforcement officers interview the drivers of all vehicles involved in an accident. However, the driving AI of an AV cannot be interviewed. Therefore, police officers need to understand what the AV was "seeing" to understand the decision that was made. To understand the perception, our recommendation is pictures to be taken from the point of view of the AV. Determine if there is any significant activity that might affect the Lidar, Radar or any other sensor of the AV. For instance weather conditions, radio equipment, or power lines could have some influence on the sensors. Moreover, to understand the decision made by the $\mathrm{AV}$, the officers may need to consider all activities in the surrounding environment that may deceive the AV. Examples of activities include actions made by pedestrians or small animals on the side of the road or on the road itself, as well as the actions of trucks or scooters or other vehicles that may move in way different than the AV.

Lastly, when accidents are caused by other humans due to their perception of an AV then it is imperative to know what the AV did to communicate its intent to the other humans in the surrounding environment. If someone perceives the AV as behaving erratically then it is as dangerous as any other erratic driver. Additionally, interviewing other drivers is of particular importance in instances where the other drivers collide with an AV even if it a minor scrape or a fender bender.

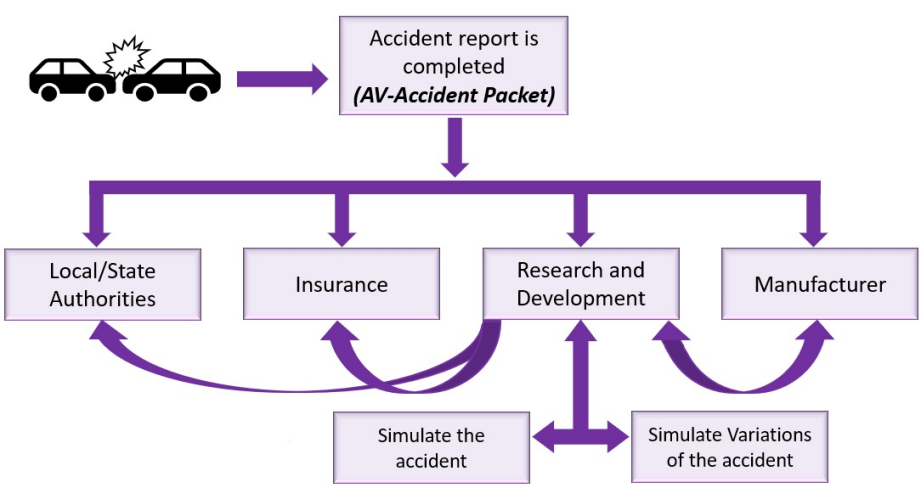

Figure 5: The accident report and the information flow

\subsection{Learning Flow}

By including the EDR recordings of the AV and environmental parameters along with the law enforcement input in the accident report, which we will call the $A V$-Accident Packet, we can analyze and understand how an accident happened and its causes. In this manner, systemic errors can be identified early and procedures can be created to escalate the issues and find remedies for it. The most critical concerns are those caused by algorithmic issues since they would be replicated across all the vehicles sharing the same software. These types of concerns need to be determined and the issues need to have a swift escalated response. On the other hand, hardware failures such as sensors or other components are also very important and can indicate reliability issues on the long term. However, hardware issues are very unlikely to be the reason behind systemic errors. Additionally, if an accident occurs where a human driver hit an AV then it is not probable that the cause requires to be escalated immediately, although the accident should be carefully considered.

To establish an overall safety model, manufactures and other involved parties must be able to utilize all the accidents' data efficiently in the production and testing of AVs. Furthermore, a database of accidents should be built and made available to be mined for possible systemic errors that wouldn't be easily identified if the data is just considered locally.

\subsection{Importance of Completed Accident reports (AV- Accident Packet)}

The rate at which automotive technology is growing is very rapid. The addition of all of this technology into the vehicles has had an affect on the way traffic is understood. Finally, the introduction of AVs in normal traffic is going to continue to change traffic behaviour which requires the reassessment of the procedures used to report accidents and how the information within the report flows (see Figure 5).

The importance of the AV-Accident Packet, as defined above, includes, but not limited to, the following main points:

- Research and Development:

- Analyzing the data to understand the causes of the accident. 
- Recreate accidents by simulation using similar scenario and extend that to include variations of parameters included (actors, road types, weather, etc.)

- Expanding the database of accidents to be used for verification and validation of AV systems.

- Manufacturers and developers

- In addition to the items listed above, the makers of AVs will have the highest interest in the performance of their product.

- Provide software updates and maybe hardware replacements.

- Insurance

- Determine Liability.

- Run risk assessments to determine the cost of insurance. For example, a company with lots of AV accidents means the cost will be higher.

\section{- Local/State Authorities}

- Collect data and analyze the statistics about accidents.

- Provide policies and regulations based on accurate information.

- Organize appropriate response in case of an accident; For instance, AVs communicate with the authorities upon the occurrence of accidents and provide an initial report.

Once the AV-Accident Packets are available, accidents can then be re-created within a simulation, and this is indispensable for multiple reasons. One is to improve the process of diagnosing errors. Also, when a correct decision is achieved which would avoid the accident or at least reduce its severity, then a validation platform, such as the one discussed in [22], can be used to check the validity of decision or action. Additionally, various test cases can be derived and simulated easily to find similar issues and fix them. Hence, this approach generates an essential feedback loop in the verification of the safety of AVs.

Presently, traditional accident data is collected and used by FARS to find systemic problems. However, this database must be enlarged to keep records and follow issues for AVs. For instance the database must include parameter measuring the reliability of sensors, conditions for edge-cases causing problems in the perception or threat recognition. It is also reasonable to have a weather index similar to the heat index of boating safety index that would give a measure on the effect of extreme weather phenomenon on the $\mathrm{AV}$ systems.

There is still a need to learn the process of building, deploying and regulating AV systems. Therefore, there is a substantial need that all parties involved must share information and thus build a system of gathering input and data which can provide a confidential way for any source to share input in order to help insure the public does not have to face unsafe conditions.

\section{Conclusion}

Stakeholders will sooner or later transform the complete picture of our roads into a fully AV-controlled environment. Undoubtedly, this promises immense range of benefits including safe transportation, to our community. Because of the differences between conventional driving and AV driving, we should treat AV accidents differently. In the AV world, the risk of systematic problems exist and it is extremely important to capture these problems at an early stage. One of the methods to capture these problems and risks is by the analysis of the current AV accidents regardless of their severity. The nature of AV systems requires capturing an incredible amount of date in order to operate (perception, decision making and action). Therefore, in order to understand the cause of an accident, a prior to the accident data must be available. In this paper, we looked at the architecture of an AV system, collected data for almost $200 \mathrm{AV}$ accidents and then we analyzed them in order to understand the root cause of these accidents. The accidents can be simulated by using the collected data to create similar scenarios of the accidents and that is vital in the verification and validation of $\mathrm{AV}$ systems.

However, with the current accident reports, some important information related to AV systems is still missing from the current accident reporting system. The current conventional automobile infrastructure must be upgraded to meet the AV needs and this paper proposes three upgrades: First, an updated law enforcement accident report that incorporates the EDR recording and sufficient details on the environment surrounding the accident. Second, a procedure for escalation which depends on the analysis of the error. Lastly, a well organized database of related AV accidents must be available to stakeholders to enable ongoing learning to find systemic issues.

\section{References}

[1] S. Sahawneh, A. J. Alnaser, M. . Akbaş, A. Sargolzaei, R. Razdan, "Requirements for the Next-Generation Autonomous Vehicle Ecosystem," in 2019 SoutheastCon, 1-6, 2019, doi:10.1109/SoutheastCon42311.2019.9020400.

[2] I. Fallon, D. O'Neill, “The world's first automobile fatality," Automobiles; Accidents; Traffic; History of medicine, 37, 601-603, 200, doi:10.1016/j.aap. 2005.02.002

[3] S. Nanda, H. Joshi, S. Khairnar, "An IOT Based Smart System for Accident Prevention and Detection," in 2018 Fourth International Conference on Computing Communication Control and Automation (ICCUBEA), 1-6, 2018, doi:10.1109/ICCUBEA.2018.8697663.

[4] S. Chandran, S. Chandrasekar, N. E. Elizabeth, "Konnect: An Internet of Things (IoT) based smart helmet for accident detection and notification," in 2016 IEEE Annual India Conference (INDICON), 1-4, IEEE, 2016, doi: 10.1109/TITS.2016.2582208.

[5] U. Alvi, M. A. K. Khattak, B. Shabir, A. W. Malik, S. R. Muhammad, "A Comprehensive Study on IoT Based Accident Detection Systems for Smart Vehicles," IEEE Access, 8, 122480-122497, 2020, doi:10.1109/ACCESS.2020.3006887.

[6] "Road traffic injuries," 2020.

[7] H. Wang, Y. Huang, A. Khajepour, Y. Zhang, Y. Rasekhipour, D. Cao, "Crash mitigation in motion planning for autonomous vehicles," IEEE transactions on intelligent transportation systems, 20(9), 3313-3323, 2019, doi: 10.1109/TRANS.2019.2582208.

[8] Y.-K. Bae, H. BENÍTEZ-SILVA, "The effects of automobile recalls on the severity of accidents," Economic Inquiry, 51(2), 1232-1250, 2013. 
[9] “Event Data Recorder," 2020.

[10] U. Bose, "The black box solution to autonomous liability," Wash. UL Rev., 92, 1325, 2014.

[11] “U.S. Federal Regulations in CFR Title 49, Subtitle B, Chapter V, Part 563," 2019.

[12] “Testing of Autonomous Vehicles with a Driver," 2019.

[13] K. Lee, D. Kum, "Collision avoidance/mitigation system: Motion planning of autonomous vehicle via predictive occupancy map," IEEE Access, 7, 5284652857, 2019, doi:10.1109/Access.2019.2582208.

[14] K. Lee, D. Kum, "Collision avoidance/mitigation system: Motion planning of autonomous vehicle via predictive occupancy map," IEEE Access, 7, 5284652857, 2019, doi:10.1109/ACCESS.2020.3005887.

[15] D. Zhao, H. Lam, H. Peng, S. Bao, D. J. LeBlanc, K. Nobukawa, C. S. Pan, "Accelerated evaluation of automated vehicles safety in lane-change scenarios based on importance sampling techniques," IEEE transactions on intelligent transportation systems, 18(3), 595-607, 2016, doi:10.1109/TITS.2016. 2582208 .
[16] F. M. Favarò, N. Nader, S. O. Eurich, M. Tripp, N. Varadaraju, "Examining accident reports involving autonomous vehicles in California," PLoS one, 12(9), e0184952, 2017.

[17] B. Schoettle, M. Sivak, "A preliminary analysis of real-world crashes involving self-driving vehicles," University of Michigan Transportation Research Institute, 2015.

[18] “PRELIMINARY REPORT, HIGHWAY, HWY19FH008," 2020.

[19] “Tesla Autopilot Car Crash Kills Driver,” 2018.

[20] “Tesla Autopilot Crashes and Causes,” 2020.

[21] “Tesla Autopilot AP1 vs AP2 vs AP3 - Differences Explained," 2021.

[22] A. J. Alnaser, M. I. Akbas, A. Sargolzaei, R. Razdan, "Autonomous vehicles scenario testing framework and model of computation," SAE International Journal of Connected and Automated Vehicles, 2(4), 2019. 